\title{
TENDENCIA EN LA DISTRIBUCIÓN ESTADÍSTICA DE LA ANOMALÍA DE LA TEMPERATURA EN CATALUÑA
}

Téllez Jurado, Beatriz

Agencia Estatal de Meteorología, Arquitecte Sert, 1, 08005 Barcelona, btellezj@aemet.es

\section{Resumen}

En este trabajo se analizan los parámetros de la función de distribución de probabilidad (FDP) de las anomalías de la temperatura máxima y mínima en Cataluña durante el periodo 1951-2017, así como su tendencia. Para ello se utilizan las series diarias de anomalías de la temperatura promediadas para Cataluña. En primer lugar, se calculan las tendencias mediante un ajuste lineal por mínimos cuadrados de las series promediadas por años. Se concluye que el aumento de la temperatura media ha sido de $0,23^{\circ} \mathrm{C}$ por década. Este valor está en línea con los obtenidos en otros estudios tanto a nivel de Cataluña como a nivel peninsular. El incremento ha sido mayor en las temperaturas máximas $\left(0,30^{\circ} \mathrm{C}\right.$ por década) que en las mínimas $\left(0,16^{\circ} \mathrm{C}\right.$ por década) y el verano ha sido la estación que más ha contribuido a este incremento $\left(0,33^{\circ} \mathrm{C}\right.$ por década). En segundo lugar, se analizan los parámetros de la FDP y su evolución. Para el análisis de los momentos de segundo orden y superior, los datos se agrupan por décadas. Es muy destacable que la varianza de las anomalías de las temperaturas máximas, especialmente en verano y en primavera, ha aumentado durante el periodo de estudio. El efecto combinado del aumento del valor medio y de la varianza ha conducido a una mayor severidad y frecuencia de los episodios extremadamente cálidos, que la que correspondería a un incremento de temperatura sin variación en su función de distribución. El sesgo en las distribuciones es siempre negativo, más acusado en las mínimas, lo que indica que los episodios extremadamente fríos tienen más peso en la distribución. La evolución de la curtosis presenta una alta variabilidad interdecadal influida por los episodios más severos, como las olas de frío de 1956 y 1985.

\section{Introducción}

El último informe de evaluación del Grupo Intergubernamental de Expertos sobre el Cambio Climático (IPCC) concluye que el calentamiento del sistema climático es inequívoco y que sus impactos se observan en todos los continentes y océanos (IPCC, 2013). El informe también afirma que, además del aumento del valor medio de la temperatura, se observan cambios en la frecuencia de valores extremos. Por otra parte, el calentamiento no es uniforme en todo el planeta y la vulnerabilidad de los seres humanos y de los ecosistemas a los eventos extremos varía de unas regiones a otras. En concordancia con las conclusiones del IPCC, en Cataluña se ha observado durante las últimas décadas un claro aumento de la temperatura media (Generalitat de Catalunya, 2016) y en repetidas ocasiones a lo largo del siglo XXI se han superado los anteriores valores máximos de las temperaturas medias anuales y estacionales. Por otra parte, diversos trabajos enmarcados en el conjunto de España o en Cataluña, basados en series iniciadas 
a mediados de siglo XX, e incluso anteriores, apuntan a un aumento asimétrico de las temperaturas máximas respecto de las mínimas, así como a un calentamiento estacional no uniforme. (Brunet et al., 2007; El Kenawy et al., 2011; Del Río, 2012; Generalitat de Catalunya, 2016).

El análisis de la evolución de los fenómenos extremos es complejo, puesto que la relación entre la temperatura media y la probabilidad de ocurrencia y severidad de episodios extremos no es lineal. En realidad, la frecuencia de extremos climáticos es más sensible a los cambios en la variabilidad que a los cambios en los valores medios, siendo mayor esta sensibilidad para los fenómenos más extremos (Katz \& Brown, 1992). Además, la combinación de cambios en el valor medio y en la dispersión lleva a una evolución asimétrica en las dos colas de la distribución: puede ocurrir que el aumento de episodios cálidos sea mucho mayor que la disminución de los fríos. Como consecuencia de todo ello, para profundizar en el estudio de la evolución de la temperatura y su reflejo en los valores extremos, es necesario estudiar no sólo la evolución del valor medio de la temperatura, sino también la evolución de los otros parámetros de la FDP. Se han publicado varios estudios en esta dirección, por ejemplo: Alexander et al. (2006) a nivel global o Klein Tank \& Können (2003) y Della-Marta et al. (2007) para Europa, obteniendo resultados diversos. Klein Tank \& Können muestran que en el periodo 1946-1975, además de un ligero enfriamiento, se produjo en Europa una reducción de la variabilidad de las temperaturas, mientras que en el periodo 1976-1999 se observó un aumento tanto en la temperatura media como en su variabilidad, esto último asociado a un estancamiento en la disminución de los episodios fríos. Por su parte, Della-Marta et al. estudian la FDP de las temperaturas máximas diarias en verano para Europa Occidental y el periodo 18802005 y encuentran un aumento tanto en la media como en la varianza.

Para llevar a cabo estos estudios en un determinado dominio geográfico es necesario construir una serie de datos diarios promediada para la zona de estudio. Entre los problemas que hay que abordar figuran la selección de estaciones, la elección del método para construir la serie y la estacionalidad intrínseca de las series de temperatura. Idealmente, debería partirse de una red de estaciones distribuidas de forma uniforme, con una resolución espacial adecuada y donde cada estación dispusiera de series continuas y homogéneas para todo el periodo de estudio. En la práctica, la densidad y topología de la red a menudo no es la idónea y las series no siempre son continuas y de alta calidad. El problema se agrava en zonas orográficamente complejas como Cataluña. Uno de los métodos utilizados para paliar el efecto de la distribución irregular de las estaciones consiste en interpolar los datos observados a una rejilla regular. De esta forma se elimina la necesidad de evaluar el peso asignado a cada serie para dar cuenta de su representatividad espacial. Esta metodología también permite aumentar la densidad de estaciones ya que elimina el requisito de continuidad de las series. En Téllez (2016) se construye la serie de anomalías diarias de Cataluña a partir de rejillas diarias de $0,005^{\circ}$ de resolución utilizando los datos de la red climatológica de AEMET. El considerable aumento en el número de datos asociado a la inclusión de series incompletas rebaja el requisito de calidad de los mismos, ya que se reduce el peso de cada valor en el producto final. En particular, se reduce el impacto de los valores atípicos ("outliers"). El aumento en el 
número de estaciones también reduce la incertidumbre asociada al método de interpolación (Burrough and McDonnell, 1998). Finalmente, en este caso, la interpolación se realiza sobre las anomalías de la temperatura respecto a sus valores de referencia, una variable que presenta menor variabilidad espacial que la propia temperatura, lo que también facilita su interpolación.

Centrar el estudio en las anomalías presenta la ventaja adicional de que se soslaya el problema de la estacionalidad inherente a las series de temperatura. Sin embargo, requiere el cálculo previo de valores de referencia diarios para todas las estaciones utilizadas. Para ello se pueden utilizar diversos métodos, pero en cualquier caso se trata de mostrar la componente no aleatoria de las variaciones diarias de la temperatura que no es captada por los valores medios mensuales (WMO, 2011). En Téllez (2016), los valores diarios de referencia para el periodo 1981-2010 se calculan ajustando los valores mensuales de referencia a una función obtenida mediante la transformada de Fourier.

\section{Datos}

Para este trabajo se utilizan tres series de base diaria conteniendo las anomalías de la temperatura máxima, mínima y media promediadas para Cataluña abarcando desde 1951 hasta 2017. Las series fueron construidas mediante la metodología descrita en Téllez (2016) a partir de los valores diarios de 400 estaciones de la red climatológica de AEMET. Integrar en el estudio todos los datos que hayan pasado los controles de calidad establecidos por el Banco Nacional de Datos Climatológico implica asumir que el conjunto de estaciones consideradas varía con el tiempo. Las anomalías diarias, referidas al periodo de referencia 1981-2010, se calcularon utilizando rejillas de 0,005 de resolución. Una interpolación bilineal y una transformada de Fourier permitieron obtener los valores de referencia diarios para cualquier estación, aun en ausencia de una serie adecuada.

Es importante tener en cuenta que los datos diarios de las series utilizadas son valores medios para Cataluña. Los resultados proporcionarán una visión general de las tendencias en este dominio geográfico, pero enmascararán fenómenos de menor escala que pueden ser localmente significativos. No hay que olvidar que en una extensión de unos $32.000 \mathrm{~km}^{2}$ las altitudes abarcan desde el nivel del mar hasta picos de más de $3000 \mathrm{~m}$ y el clima presenta una gran diversidad, con amplias zonas mediterráneas conviviendo con otras donde dominan las influencias atlánticas.

\section{Metodología}

El análisis de los parámetros de la distribución estadística de las anomalías térmicas diarias, así como de su evolución temporal, se ha realizado mediante técnicas estadísticas simples. Para estudiar la tendencia del valor medio de las tres series, los valores diarios se han promediado por años y estaciones. Las nuevas series obtenidas han permitido calcular las tendencias mediante un ajuste lineal por mínimos cuadrados.

Los parámetros de las FDP (mínimo, máximo, media, mediana, varianza, sesgo, curtosis, percentil 10 y percentil 90) se han calculado a partir de las series iniciales, de base diaria. Sin embargo, para analizar la evolución temporal de los momentos de orden superior, las anomalías se han promediado de nuevo, en este caso por décadas. Esta 
agrupación elimina las variaciones interanuales bruscas y atenúa el efecto del comportamiento no aleatorio de la temperatura, es decir, el hecho de que la temperatura de un día -o su anomalía- sea fuertemente dependiente del valor correspondiente al día anterior.

\section{Resultados y discusión}

\subsection{Tendencia del valor medio anual y estacional de la temperatura}

Las series de valores medios anuales de las anomalías de las temperaturas máximas y mínimas muestran un comportamiento creciente. (Figura 1). El ajuste lineal por mínimos cuadrados cuantifica el calentamiento en $0,23^{\circ} \mathrm{C}$ /década para la temperatura media diaria durante el periodo 1951-2017. El coeficiente de determinación $\left(r^{2}\right)$ del ajuste es de 0,47. Las temperaturas máximas han contribuido más al calentamiento que las mínimas, con una tendencia de $0,30{ }^{\circ} \mathrm{C} /$ década frente a $0,16^{\circ} \mathrm{C} /$ década. Estos resultados son muy similares a los obtenidos en Generalitat de Catalunya (2016), también para el dominio geográfico de Cataluña y para un periodo muy similar. Por otra parte, el aumento de temperatura no ha sido uniforme durante todo el periodo de estudio. Como se verá más adelante con mayor detalle, se puede identificar una primera fase más fría, que culmina en la década de 1970 y una segunda fase más cálida, que se prolonga hasta el final del periodo de estudio.

Fig. 1.

Evolución de las anomalías de las temperaturas máximas (izquierda) y mínimas (derecha) diarias durante el periodo 1951-2017. Las
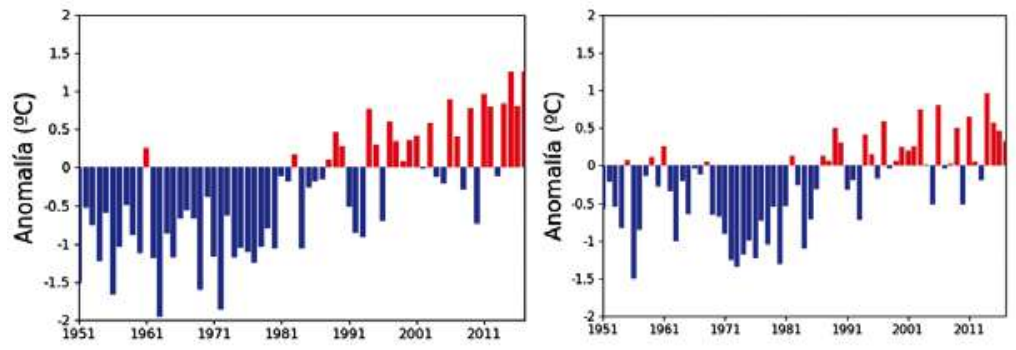
anomalías están calculadas en base al periodo de referencia 1981-2010

De la misma forma que las tendencias no han sido uniformes a lo largo del periodo de estudio, tampoco lo han sido en las cuatro estaciones del año. Como se detalla en la tabla 1, el verano ha sido la que más ha contribuido al calentamiento, seguido a bastante distancia por la primavera. Estos resultados también están en línea con los obtenidos por Generalitat de Catalunya (2016). Finalmente, puede destacarse que el patrón asimétrico del aumento de las temperaturas máximas respecto al de las mínimas se repite en todas las estaciones del año.

\begin{tabular}{|lccccc|}
\hline & Invierno & Primavera & Verano & Otoño & Año \\
\hline T máxima & $0,24(0,21)$ & $0,29(0,23)$ & $0,43(0,45)$ & $0,25(0,22)$ & $0,30(0,55)$ \\
\hline T mínima & $0,15(0,07)$ & $0,15(0,13)$ & $0,23(0,29)$ & $0,15(0,10)$ & $0,16(0,30)$ \\
\hline T media & $0,19(0,15)$ & $0,22(0,21)$ & $0,33(0,41)$ & $0,20(0,18)$ & $0,23(0,47)$ \\
\hline
\end{tabular}

Tabla 1. Tendencia de la temperatura máxima, mínima y media $\left({ }^{\circ} \mathrm{C} /\right.$ década) para todo el año y por estaciones durante el periodo 1951-2017. Entre paréntesis se indica el coeficiente de determinación 


\subsection{Parámetros de las FDP de las series diarias de anomalía de la temperatura}

Las series diarias de anomalías de las temperaturas máximas y mínimas para el periodo de estudio 1951-2017 presentan una distribución cercana a la normal, como puede apreciarse en la figura 2 , al igual que las series diarias agrupadas por estaciones. Los parámetros de las FDP de estas series anuales y estacionales (valores máximo y mí-

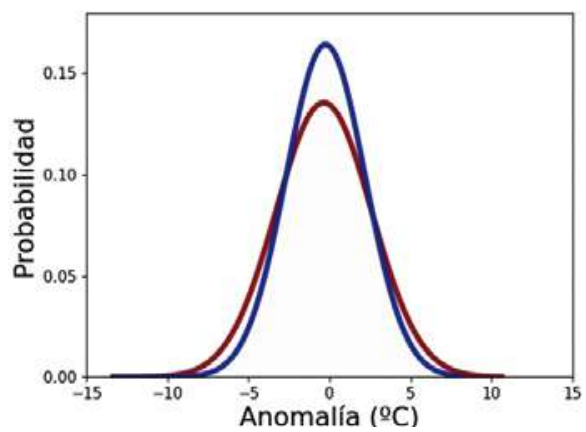
nimo, media, mediana, varianza, sesgo, curtosis, percentil 10 y percentil 90) se detallan en la tabla 2 para las temperaturas máximas y en la tabla 3 para las mínimas.

Fig. 2. Funciones de distribución de probabilidad de las series diarias de anomalías de temperatura máxima (rojo) y mínima (azul) para el periodo 1951-2017

Mínimo $\left({ }^{\circ} \mathrm{C}\right)$ Máximo $\left({ }^{\circ} \mathrm{C}\right)$ Media $\left({ }^{\circ} \mathrm{C}\right)$ Mediana $\left({ }^{\circ} \mathrm{C}\right)$ Varianza $\left({ }^{\circ} \mathrm{C} 2\right)$ Sesgo Curtosis Perc. $10\left({ }^{\circ} \mathrm{C}\right)$ Perc. $90\left({ }^{\circ} \mathrm{C}\right)$

$\begin{array}{lccccccccr}\text { Invierno } & -13,34 & 7,96 & -0,38 & -0,22 & 8,19 & -0,42 & 0,62 & -4,05 & 3,14 \\ \text { Primavera } & -11,62 & 10,63 & -0,20 & -0,19 & 10,68 & -0,05 & -0,21 & -4,44 & 4,01 \\ \text { Verano } & -12,72 & 9,07 & -0,52 & -0,38 & 8,30 & -0,13 & 0,04 & -4,27 & 3,05 \\ \text { Otoño } & -10,92 & 9,05 & -0,32 & -0,19 & 7,49 & -0,13 & 0,00 & -3,91 & 3,03 \\ \text { Anual } & -13,34 & 10,63 & -0,35 & -0,25 & 8,68 & -0,16 & 0,13 & -4,18 & 3,31\end{array}$

Tabla 2. Parámetros de las FDP estacionales y anual de las anomalías de la temperatura máxima

\section{Mínimo $\left({ }^{\circ} \mathrm{C}\right)$ Máximo $\left({ }^{\circ} \mathrm{C}\right)$ Media $\left({ }^{\circ} \mathrm{C}\right)$ Mediana $\left({ }^{\circ} \mathrm{C}\right)$ Varianza $\left({ }^{\circ} \mathrm{C} 2\right)$ Sesgo Curtosis Perc. $10\left({ }^{\circ} \mathrm{C}\right)$ Perc. $90\left({ }^{\circ} \mathrm{C}\right.$}

$\begin{array}{llllllllll}\text { Invierno } & -13,34 & 8,21 & -0,29 & -0,18 & 7,96 & -0,36 & 0,56 & -3,83 & 3,22 \\ \text { Primavera } & -10,11 & 7,84 & -0,10 & 0,02 & 5,39 & -0,25 & -0,03 & -3,25 & 2,80 \\ \text { Verano } & -7,60 & 5,91 & -0,27 & -0.10 & 3,70 & -0,40 & 0,12 & -2,88 & 2,04 \\ \text { Otoño } & -9,63 & 8,21 & -0,30 & -0,18 & 6,62 & -0,20 & -0,17 & -3,73 & 2,88 \\ \text { Anual } & -13,34 & 8,21 & -0,24 & -0,10 & 5,91 & -0,31 & 0,42 & -3,43 & 2,72\end{array}$

Tabla 3. Parámetros de las FDP estacionales y anual de las anomalías de la temperatura mínima

Una primera inspección de los resultados muestra que la dispersión de las anomalías es mayor para las temperaturas máximas que para las mínimas. Los rangos de variación no difieren excesivamente: las anomalías de las temperaturas diurnas se extienden entre valores inferiores a $-13^{\circ} \mathrm{C}$ y superiores a $10^{\circ} \mathrm{C}$ y las de las nocturnas entre $-13^{\circ} \mathrm{Cy} 8^{\circ} \mathrm{C}$. No obstante, los valores que definen estos rangos son puntuales y son los valores de las varianzas de ambas distribuciones $\left(8,68^{\circ} \mathrm{C}^{2}\right.$ para las máximas frente a $5,91^{\circ} \mathrm{C}^{2}$ para las mínimas $)$ los que confirman que la variabilidad de las temperaturas diurnas es más alta y, por tanto, su comportamiento es más dispar. Los percentiles 10 y 90 de ambas distribuciones corroboran que la severidad de los episodios extremos es mayor para las temperaturas máximas 
que para las mínimas. A escala estacional se repite el mismo patrón. En particular, se puede destacar la gran variabilidad de las anomalías de las temperaturas diurnas de primavera (varianza de $10,68^{\circ} \mathrm{C}^{2}$ ) frente al acusado agrupamiento en torno a los valores medios que se produce en las noches estivales (varianza de $3,7^{\circ} \mathrm{C}^{2}$ ).

Las dos FDP están sesgadas hacia los valores negativos. La asimetría se confirma por la diferencia positiva entre la mediana y la media de las dos distribuciones. El valor negativo del sesgo es un indicador del desequilibrio existente entre los episodios fríos y los cálidos. La asimetría es más acusada para las temperaturas mínimas (sesgo de -0,31) que para las máximas $(-0,16)$. Esta característica se mantiene en todas las estaciones excepto en el invierno. Las temperaturas máximas invernales son las que presentan una mayor descompensación entre episodios fríos y cálidos, en contraste con los días primaverales, que presentan una distribución cuasi-simétrica. El mayor sesgo en las temperaturas mínimas se da tanto en verano como en invierno, siendo bastante superior al de las estaciones intermedias.

Finalmente, las dos series anuales presentan una FDP con curtosis positiva. Siguiendo la interpretación de Westfall (2014), ello indicaría que estas series tienen mayor propensión que una distribución normal a presentar valores muy extremos, atípicos. Con diferencia, la estación que más contribuye al cómputo total de este parámetro es el invierno, donde quedan reflejados los extraordinarios episodios fríos de febrero de 1956 y enero de 1985, tanto en las temperaturas máximas como en las mínimas.

\subsection{Evolución de la función de distribución de probabilidad de las series diarias de anomalía de la temperatura}

El estudio de la evolución de la FDP de las series, así como de los parámetros que las definen, requiere que los valores de dichas series se agrupen para distintos intervalos de tiempo. En el caso de la media (apartado 4.1) los datos se promediaban por años. Para los momentos de orden superior, los datos se han promediado por décadas. Esta agrupación elimina las variaciones interanuales bruscas y atenúa el efecto del comportamiento no aleatorio de la temperatura, es decir, el hecho de que la temperatura de un día -o su anomalía- sea fuertemente dependiente del valor correspondiente al día anterior. En la figura 3 se muestra la evolución decadal de la FDP, en la figura 4 la evolución de sus parámetros y en la figura 5 la evolución decadal de las FDP de cada estación.
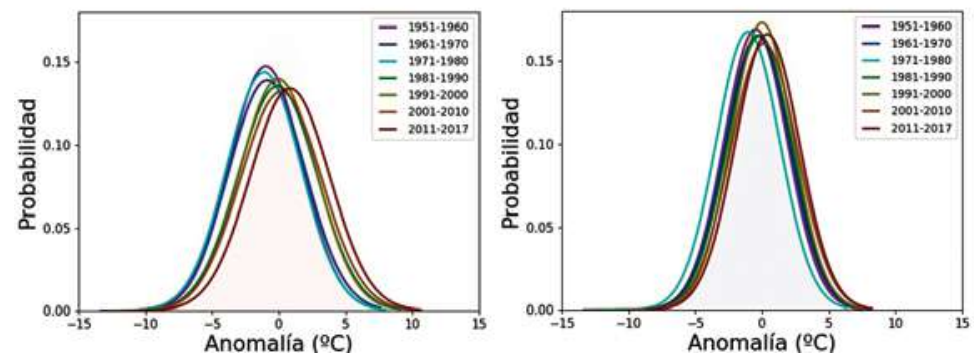

Fig. 3. Evolución decadal de la FDP de las series de anomalías de las temperaturas máximas (izquierda) y mínimas (derecha) 
De manera similar a la evolución descrita en el apartado 4.1, los valores medios de las anomalías de las distribuciones decadales muestran un comportamiento creciente pero no monótono. La evolución de las anomalías de las temperaturas máximas y mínimas es distinta, siendo mayor el crecimiento de las primeras. (Figura 4). Destacan los cambios bruscos entre las décadas de 1960 y 1980, con un enfriamiento seguido de un abrupto calentamiento. También destaca el aumento paulatino de las décadas posteriores (1980, 1990, 2000), alcanzando en la última fase valores medios ligeramente superiores a los de referencia. Por último, durante los últimos años de estudio (2011-2017), un calentamiento más acusado que en las décadas anteriores, especialmente para las temperaturas máximas, provoca que las anomalías alcancen los valores más altos de todo el periodo. El enfriamiento observado en la década de 1970 y el abrupto calentamiento posterior está en línea con otros estudios enmarcados en España (Brunet et al., 2007). Lo mismo ocurre con la ralentización aparente del aumento de temperaturas durante el periodo 1990-2010 (“hiatus") (Gonzalez-Hidalgo, 2016, Serrano, 2017)
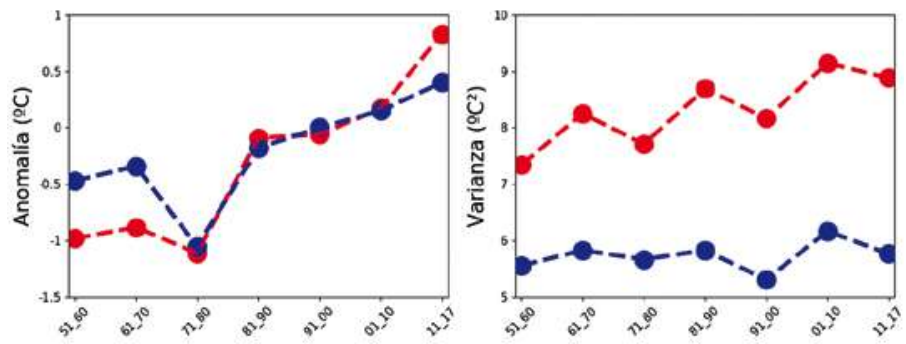

Fig. 4. Evolución decadal de los parámetros de la FDP de las series de anomalías de las temperaturas
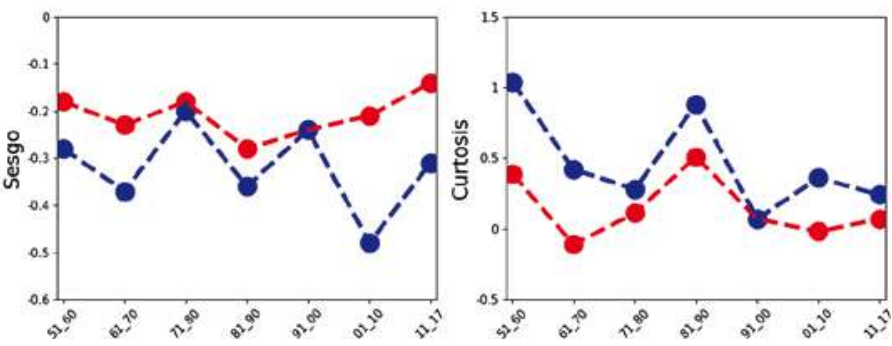
máximas (roja) y mínimas (azul). Arriba: media (izquierda) y varianza (derecha). Abajo: sesgo (izquierda) y curtosis (derecha)

En el apartado anterior se veía que la varianza de las temperaturas máximas era mayor que la de las mínimas. Esta característica se repite en todas las décadas del estudio. Sin embargo, la evolución interdecadal no ha sido la misma en los dos casos. Mientras que en las anomalías de la temperatura máxima se observa un aumento de la varianza, en las anomalías de la temperatura mínima la varianza se ha mantenido con valores bastante constantes. (Figura 4). Por estaciones, destaca el aumento de variabilidad que se ha observado en las anomalías de las temperaturas máximas de verano y primavera. La variabilidad ha aumentado también en las mínimas estivales, pero no de forma tan acusada como en las máximas. El aumento del valor medio y de la varianza en las anomalías de 
las temperaturas máximas de verano y primavera y en las mínimas estivales ha quedado reflejado en la evolución de las FDP decadales como un desplazamiento hacia los valores más cálidos y un ensanchamiento progresivo de la distribución (Figura 5). Ambas alteraciones suman sus efectos sobre la cola cálida de la distribución, dando como resultado no sólo un aumento de severidad de los episodios extremadamente cálidos, sino también un mayor aumento de la probabilidad de ocurrencia de estos episodios. La asimetría en la evolución de las colas frías y cálidas de la FDP ha provocado un mayor aumento de episodios cálidos que disminución de los fríos.
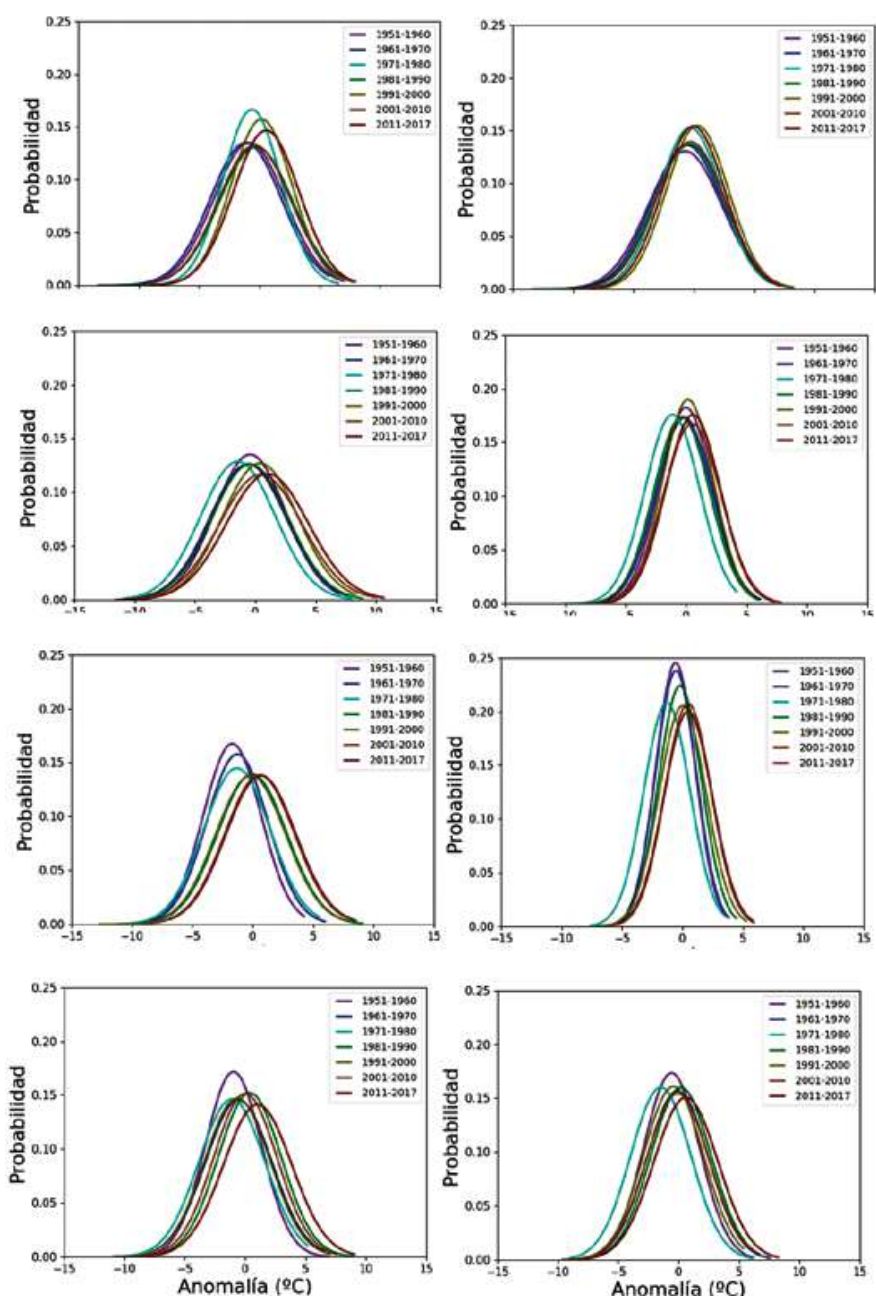

La variabilidad interdecadal del sesgo y de la curtosis es muy grande y no resulta fácil alcanzar conclusiones sólidas en cuanto a la tendencia. Se puede destacar un fuerte sesgo negativo en las temperaturas mínimas durante la década de 2000. Se trataría de la distribución que más descompensación presenta entre los episodios extremos fríos y cálidos. En cuanto a la curtosis, muy influenciada por los episodios muy extremos, los mayores picos se dieron en las décadas de 1950 y 1980 , asociados a los episodios extremadamente fríos de 1956 y 1985.

Fig. 5. Evolución decadal de las FDP estacionales. En el lado izquierdo, anomalías de las temperaturas máximas y en el derecho, anomalías de las temperaturas mínimas. De arriba abajo: invierno, primavera, verano y otoño. 


\section{Conclusiones}

Este artículo presenta un estudio de la distribución de probabilidad de las anomalías térmicas de Cataluña durante el periodo 1951-2017. El estudio se aborda mediante el análisis de las anomalías diarias promediadas para Cataluña y referidas al periodo 19812010, reflejando una visión general de las tendencias en este dominio geográfico, aunque los fenómenos de menor escala, que pueden ser localmente significativos, quedarán enmascarados.

La evolución de la temperatura media en el periodo de estudio presenta un comportamiento creciente pero no monótono. El calentamiento calculado es de $0,23^{\circ} \mathrm{C} /$ década para la temperatura media diaria, siendo mayor la contribución de las temperaturas máximas que la de las mínimas. Por estaciones, el verano y después la primavera son las que presentan un aumento de temperatura más acusado.

Las FDP de las series diarias de anomalías de temperatura presentan distribuciones cercanas a la normal, con una mayor dispersión $\left(8,68^{\circ} \mathrm{C}^{2}\right)$ en las anomalías de las temperaturas máximas que en las de las mínimas $\left(5,91^{\circ} \mathrm{C}^{2}\right)$. Las dos FDP están sesgadas hacia los valores negativos, lo que indica que hay un desequilibrio entre los episodios fríos y los cálidos. Finalmente, las dos series presentan una curtosis positiva, lo que indicaría que tienen mayor propensión que una distribución normal a generar valores muy extremos.

Finalmente, la evolución interdecadal de las FDP de las series muestra un aumento de la varianza para las anomalías de las temperaturas máximas frente a un comportamiento más constante para las mínimas. Por estaciones, destaca el aumento de variabilidad que se ha observado en las temperaturas máximas de verano y primavera. Este aumento, aunque menos acusado, también se ha encontrado en las mínimas estivales.

El aumento combinado del valor medio y de la varianza provoca un aumento en la ocurrencia e intensidad de episodios extremadamente cálidos. Este aumento es mayor que si la temperatura media hubiera aumentado sin cambios en la varianza. En paralelo con este mayor incremento en los episodios extremadamente cálidos, se produce una disminución más lenta en la ocurrencia e intensidad de los fenómenos extremadamente fríos.

\section{Agradecimientos}

Mi agradecimiento a Enric Terradellas, Cruz Casas y Oscar Criado de la Delegación Territorial de Aemet de Cataluña, por su apoyo y su ayuda para solucionar las dudas y los problemas que han ido surgiendo al elaborar este estudio.

\section{Referencias}

- Alexander, L. V., Zhang, X., Peterson, T. C., Caesar, J., Gleason, B., Klein Tank, A. M. G., ... \& Tagipour, A., 2006: Global observed changes in daily climate extremes of temperature and precipitation. Journal of Geophysical Research: Atmospheres, 111(D5).

- Brunet, M., Jones, P. D., Sigró, J., Saladié, O., Aguilar, E., Moberg, A., ... \& López, D., 2007: Temporal and spatial temperature variability and change over Spain during 1850-2005. Journal of Geophysical Research: Atmospheres, 112(D12). 
Burrough, P. A., McDonnell, R. A., \& Lloyd, C. D., 2015: Principles of geographical information systems. Oxford University Press. $352 \mathrm{pp}$.

- Del Río, S., Cano-ortiz, A., Herrero, L., \& Penas, A., 2012: Recent trends in mean maximum and minimum air temperatures over Spain (1961-2006). Theoretical and Applied Climatology 109(3-4), 605-626. doi:10.1007/s00704-012-0593-2

- Della-Marta, P. M., Haylock, M. R., Luterbacher, J., \& Wanner, H., 2007: Doubled length of western European summer heat waves since 1880. Journal of Geophysical Research: Atmospheres, 112(D15).

- El Kenawy, A., López-Moreno, J. I., \& Vicente-Serrano, S. M. 2011:. Recent trends in daily temperature extremes over northeastern Spain (1960-2006). Natural Hazards and Earth System Sciences, 11(9), 2583.

- Generalitat de Catalunya, 2016: El canvi climàtic a Catalunya: Tercer informe sobre el canvi climàtic a Catalunya. Generalitat de Catalunya and Institut d'Estudis Catalans, Barcelona, 625 pp.

- Gonzalez-Hidalgo, J. C., Peña-Angulo, D., Brunetti, M., \& Cortesi, N., 2016: Recent trend in temperature evolution in Spanish mainland (1951-2010): from warming to hiatus. International Journal of Climatology, 36(6), 2405-2416.

- IPCC, 2013: Climate Change 2013: The Physical Science Basis. Contribution of Working Group I to the Fifth Assessment Report of the Intergovernmental Panel on Climate Change. Cambridge University Press, Cambridge, United Kingdom and New York, NY, USA, 1535 pp., doi:10.1017/ CBO9781107415324

- Katz, R. W., \& Brown, B. G. 1992: Extreme events in a changing climate: variability is more important than averages. Climatic change, 21(3), 289-302.

- Klein Tank, A. M. G., \& Können, G. P., 2003: Trends in indices of daily temperature and precipitation extremes in Europe, 1946-99. Journal of climate, 16(22), 3665-3680.

- Serrano, S. M. V., Camino, E. R., Castro, F. D., El Kenawy, A. M., \& Molina, C. A., 2017: An updated review on recent trends in observational surface atmospheric variables and their extremes over Spain. Cuadernos de investigación geográfica, (43), 209-232.

- Téllez, B., 2016: Seguimiento de las anomalías diarias de la temperatura media de Cataluña. XXXIV Jornadas Cientificas de la AME, Teruel. ISBN 978-84-617-5240-9

- Westfall, P. H. 2014: Kurtosis as peakedness, 1905-2014. RIP. The American Statistician, 68(3), 191-195.

- WMO, 2011: Guía de prácticas climatológicas. OMM núm. 100. Organización Meteorológica Mundial, 128 pp., ISBN 978-92-63-30100-0. 